\title{
Different Contributions of the Human Amygdala and Ventromedial Prefrontal Cortex to Decision-Making
}

\author{
Antoine Bechara, ${ }^{1,2}$ Hanna Damasio, ${ }^{1,3}$ Antonio R. Damasio, ${ }^{1,3}$ and Gregory P. Lee ${ }^{4}$ \\ Departments of ${ }^{1}$ Neurology and ${ }^{2}$ Anatomy and Cell Biology, University of lowa College of Medicine, lowa City, lowa \\ 52242, ${ }^{3}$ The Salk Institute of Biological Studies, La Jolla, California 92186, and 4Section of Neurosurgery, Medical College \\ of Georgia, Augusta, Georgia 30912
}

The somatic marker hypothesis proposes that decision-making is a process that depends on emotion. Studies have shown that damage of the ventromedial prefrontal (VMF) cortex precludes the ability to use somatic (emotional) signals that are necessary for guiding decisions in the advantageous direction. However, given the role of the amygdala in emotional processing, we asked whether amygdala damage also would interfere with decision-making. Furthermore, we asked whether there might be a difference between the roles that the amygdala and VMF cortex play in decision-making. To address these two questions, we studied a group of patients with bilateral amygdala, but not VMF, damage and a group of patients with bilateral VMF, but not amygdala, damage. We used the "gambling task" to measure decision-making performance and electrodermal activity (skin conductance responses, SCR) as an index of so-

Recent studies have focused on the role of the ventromedial prefrontal (VMF) cortex in the activation of somatic states that influence decision-making (Bechara et al., 1996, 1997a; Damasio, 1996). Although the somatic marker hypothesis proposes that both the VMF cortex and the amygdala are components of a neural system necessary for implementing advantageous decisions (Damasio, 1994), the role of the amygdala in the process has not been tested yet. Therefore, the objectives of this study were (1) to test whether amygdala damage interferes with the process of decision-making and (2) to test whether the amygdala and the VMF cortex play different roles in the process.

To measure decision-making, we used the gambling task, a paradigm designed to simulate real-life decisions in terms of uncertainty, reward, and punishment (Bechara et al., 1994). In the gambling task the subjects have to choose between decks of cards that yield high immediate gain but larger future loss, i.e., longterm loss, and decks that yield lower immediate gain but a smaller future loss, i.e., a long-term gain. Skin conductance responses (SCRs) are used as an index of somatic state activation. In previous studies we showed that choosing advantageously in the gambling task is a correlate of the development of anticipatory SCRs, which normal subjects begin to generate before choosing

Received Jan. 29, 1999; revised April 2, 1999; accepted April 12, 1999.

This work was supported by National Institute of Neurological Diseases and Stroke Grant PO1 NS19632. We are indebted to the valuable contribution of Jon Spradling for computerizing the gambling task used in this study. Also, we are indebted to the contribution of Denise Krutzfeldt for scheduling the subjects and of Andrea Hindes for testing some of the subjects in this study.

Correspondence should be addressed to Dr. Antoine Bechara, Department of Neurology, University of Iowa Hospitals and Clinics, Iowa City, IA 52242.

Copyright (C) 1999 Society for Neuroscience $0270-6474 / 99 / 195473-09 \$ 05.00 / 0$ matic state activation. All patients, those with amygdala damage as well as those with VMF damage, were (1) impaired on the gambling task and (2) unable to develop anticipatory SCRs while they pondered risky choices. However, VMF patients were able to generate SCRs when they received a reward or a punishment (play money), whereas amygdala patients failed to do so. In a Pavlovian conditioning experiment the VMF patients acquired a conditioned SCR to visual stimuli paired with an aversive loud sound, whereas amygdala patients failed to do so. The results suggest that amygdala damage is associated with impairment in decision-making and that the roles played by the amygdala and VMF in decision-making are different.

Key words: decision-making; conditioning; gambling task; skin conductance; emotion; amygdala; prefrontal cortex

from a risky deck (Bechara et al., 1996, 1997a). Patients with VMF cortex lesions choose disadvantageously in this task, and their behavior is in fact a correlate of their failure to acquire anticipatory SCRs (Bechara et al., 1996, 1997a). Our first hypothesis is that the amygdala is also a critical structure in a neural system necessary for somatic state activation and for implementing advantageous decisions. We predict that patients with bilateral amygdala damage will be similar to VMF patients in terms of (1) choosing disadvantageously on the gambling task and (2) failing to develop anticipatory SCRs before selecting a disadvantageous response.

Our second hypothesis is that the poor decision-making after damage to the amygdala or VMF cortex is the consequence of different kinds of impairment. The decision-making impairment after amygdala damage is possibly the indirect consequence of the patients' inability to experience sufficiently the emotional attributes of a situation that is charged with emotion, therefore precluding the possibility to evoke somatic states after winning or losing money and thus precluding the enactment of a somatic state when deliberating a decision with future consequences. On the other hand, the decision-making impairment after VMF damage is related to an inability to integrate effectively all of the somatic state information triggered by the amygdala as well as other somatic effectors such as the hypothalamus and brainstem nuclei. When a normal subject is faced with a decision to select a card from a specific deck, the neural activity pertaining to this information is signaled to VMF cortices, which in turn activate the amygdala. This latter activity would reconstitute a somatic state that integrates the numerous and conflicting instances of reward and punishment related to that deck. The final somatic 
state, indexed by anticipatory SCRs, then would influence the decision to select from, or avoid, that deck. VMF damage precludes this process. Therefore, we predicted that the amygdala patients would fail to generate SCRs when they win or lose play money in the gambling task. By contrast, the VMF patients would generate SCRs after winning or losing.

We conducted a further experiment to test the hypothesis that amygdala, but not VMF, damage precludes or diminishes the ability of subjects to evoke the emotional attribute of an emotionally charged stimulus. We tested the ability of subjects to acquire SCRs to visual stimuli that had been paired repeatedly with an aversive loud sound. Consistent with previous studies (Bechara et al., 1995; LaBar et al., 1995, 1998), we predicted that the patients with amygdala, but not VMF, damage would fail to evoke SCRs when viewing the visual stimuli paired with an aversive sound.

\section{MATERIALS AND METHODS}

Subjects. We studied 13 normal subjects as a control group (seven women and six men; age range from 22 to 58 years with 7-16 years of education) and a brain-damaged group with 10 subjects, which included five amygdala patients (one woman and four men) and five VMF patients (three women and two men). The patients in the brain-damaged groups had an age range from 19 to 58 years with $8-18$ years years of education, a verbal I.Q. between 86 and 126, and a performance I.Q. between 88 and 116 .

Anatomical analyses. Anatomical analyses were performed on raw data from high-resolution magnetic resonance scans and x-ray-computerized tomograms, using the standard procedures of the Division's Laboratory of Neuroimaging and Human Neuroanatomy. These include both template plotting (Damasio and Damasio, 1989) and three-dimensional volume reconstruction, using Brainvox (Damasio and Frank, 1992; Frank et al., 1997). All lesions at the time of testing and anatomical analyses were in the chronic stage and stable. The amygdala patients R.L. and D.B. have had previous anatomical analyses (Lee et al., 1988a,b, 1995).

The gambling task. We used a computerized version of the gambling task based on the original as described in Bechara et al. (1994). An automated and computerized method for collecting, measuring, and analyzing SCR data was used instead of the earlier described methods (Bechara et al., 1994, 1996, 1997a, 1998).

In the computerized version of the gambling task, the subject sees the four decks of cards on a computer screen. The decks are labeled A, B, C, and $\mathrm{D}$ at the top end of each deck. Using a mouse, the subject can click on a card on any of the four decks. The computer tracks the sequence of the cards selected from the various decks. Every time the subject clicks on a deck "to pick a card," the computer generates a distinct sound (similar to a casino slot machine). The face of the card appears on top of the deck (the color is either red or black), and a message is displayed on the screen indicating the amount of money the subject has won or lost. On the top of the computer screen is a green bar that changes according to the amount of money won or lost after each selection. A gain is indicated by a proportionate increase in the length of the green bar, and a loss is indicated by a proportionate decrease in the bar length. Once the money is added or subtracted, the face of the card disappears, and the subject can select another card.

The intertrial interval between making two consecutive card selections can be set by the examiner at the beginning of the task. The total number of card selections (trials) in the experiment also is set at the beginning. In the present experiment we set the intertrial interval at $6 \mathrm{sec}$ to allow for psychophysiological recordings (see below). The total number of trials was set at 100 card selections. The experiment shuts off automatically when the 100 selection trials are complete. However, as in previous experiments, the subject was not told in advance how many cards he/she was going to pick. To score the performance of the subject on the gambling task, we added the number of cards picked from decks A and B in each block of 20 cards; we added the number of cards picked from decks $\mathrm{C}$ and $\mathrm{D}$ separately in each block of 20 cards.

Each deck of cards is programmed to have 40 cards: 20 of the cards have a black face and 20 have a red face. The backs of the cards as they appear on the screen all look the same, like real decks of cards. The sequence of red and black cards in each deck and the gains and losses for each card selection are based on the original version of this task (Bechara et al., 1994). In brief, every 10 cards from deck A over the course of trials gain $\$ 1000$, but there are also five unpredictable punishments ranging from $\$ 150$ to $\$ 350$, bringing the total loss to $\$ 1250$. Every 10 cards from deck B gain $\$ 1000$, but there is also one big punishment for $\$ 1250$. On the other hand, every 10 cards from deck C or D amount only to a gain of $\$ 500$, but the losses are also smaller, i.e., $\$ 250$ (ranging from $\$ 25$ to $\$ 75$ in deck $\mathrm{C}$ and one $\$ 250$ loss in deck D), bringing a net gain of $\$ 250$. In summary, decks $\mathrm{A}$ and $\mathrm{B}$ are equivalent in terms of overall net loss over trials. Similarly, decks C and D are equivalent in terms of overall net gains. The difference is that decks $\mathrm{A}$ and $\mathrm{C}$ have higher frequency but lower magnitude punishment. Decks B and D have lower frequency but higher magnitude punishment. Thus, decks $\mathrm{A}$ and $\mathrm{B}$ are disadvantageous because they cost more in the long run. Decks $\mathrm{C}$ and $\mathrm{D}$ are advantageous because they result in an overall gain in the long run.

In Figure 1 of Bechara et al. (1994), each square on the score sheet represents a card in a deck. Each square that has a " 0 " or "a negative number" corresponds to a red card, and each square without any marking corresponds to a black card. The computer displays a $\$ 100$ reward every time the subject picks a card from deck A or B and displays $\$ 50$ when the choice is from deck $\mathrm{C}$ or $\mathrm{D}$. When a card corresponding to a square with a "negative number" is picked, the computer displays a message: "... You have won X dollars, but you also have lost Y dollars..." (the Y amount corresponds to the negative number inside the square), and the net loss is reflected automatically on the green bar on the screen. When a card corresponding to a square that is blank or with " 0 " is picked, the subject wins, and there is no loss. The computer displays the following message: "... You have won X dollars..."; the gain is also reflected on the green bar.

We note that the gambling task involves 100 selections of cards, and there are only 40 cards in each deck. Thus, it is possible to run out of cards from a given deck. When a given deck runs out of cards, the subject is instructed to stop picking from that deck and continue choosing from the remaining decks. In reality, this situation arises very seldom. The reason is that the task is more difficult than it appears to be. It is difficult for subjects to be sure whether to pick constantly from a given deck. Therefore, their selections are distributed among the different decks, and the decks seldom run out of cards.

In summary, after clicking to turn each card, the subject receives some money (the amount is displayed on the screen). On some cards the subject both wins money and pays a penalty (the amounts are displayed on the screen). Clicking to turn any card from deck A or deck B yields $\$ 100$; turning any card from deck C or deck D yields $\$ 50$. However, the ultimate future yield of each deck varies because the penalty amounts are higher in the high-paying decks (A and B), leading to a negative balance, and lower in the low-paying decks (C and $\mathrm{D})$, leading to a final gain. Thus, decks A and B are "disadvantageous," whereas decks C and D are "advantageous."

So that they can perform the task, the subjects are given the following verbal instructions:

1. In front of you on the screen, there are four decks of cards A, B, C, and $\mathrm{D}$.

2. I want you to select one card at a time, by clicking on the card, from any deck you choose.

3. Each time you select a card from a deck, the color of the card turns red or black, and the computer will tell you that you won some money. I won't tell you how much money you will win. You will find out along the way. Every time you win, the green bar gets longer.

4. Every so often, however, when you click on a card, the computer tells you that you won some money, but then it says that you also lost some money. I won't tell you when you will lose or how much you will lose. You will find out along the way. Every time you lose, the green bar gets shorter.

5. You are absolutely free to switch from one deck to another any time you wish.

6. The goal of the game is to win as much money as possible and, if you find yourself unable to win, make sure you avoid losing money as much as possible.

7. I won't tell you for how long the game will continue. You must keep on playing until the computer stops.

8. You will get this $\$ 2000$ credit (see the green bar) to start the game. At the end, we will see how much you won or lost. The red bar here is a reminder of how much money you borrowed to play the game.

9. It is important to know that the colors of the cards are irrelevant in this game. The computer does not make you lose money at random. However, there is no way for you to figure out when the computer will make you lose. All I can say is that you may find yourself losing money 
on all of the decks, but some decks will make you lose more than others. You can win if you stay away from the worst decks.

$S C R$ recording during the gambling task. Electrodes are attached to the thenar and hypothenar areas on the palms after the subject is seated in a comfortable chair in front of the computer screen. As the subject performs the task, SCR activity is recorded continuously and collected simultaneously on a Macintosh computer. Each time the subject clicks the mouse and selects a card, this action is recorded as a "mark" on the polygram of SCR activity. Each click is registered as a selection from the specific deck that was chosen. Thus, SCRs generated in association with a specific card from a specific deck can be identified precisely on the polygram. Although the intertrial interval is set at $6 \mathrm{sec}$, in reality the time interval between two card selections is longer, because it takes a few additional seconds for the subject to decide which card to pick next. This time interval varies from trial to trial. It is on average $\sim 10 \mathrm{sec}$. During the $6 \mathrm{sec}$ intertrial interval the decks are displayed continuously on the screen, and the subject can ponder which deck to choose next. However, if the subject clicks the mouse to select a card during that time interval, the computer will not respond, and therefore no record is generated.

The SCRs generated during the task are divided into three categories: (1) reward SCRs, which are generated after turning cards for which there is a reward and no penalty; (2) punishment SCRs, which are generated after turning a card for which there is a reward and an immediate penalty; (3) anticipatory SCRs, which are generated previous to turning a card from any given deck, i.e., during the time period the subject ponders from which deck to choose. The time windows for the reward and punishment SCRs are the $5 \mathrm{sec}$ immediately after the click of a card. SCRs generated during the end of the reward/punishment window and before the next click of a card are considered anticipatory SCRs. The current procedure of scoring these SCRs is automated. The SCR data were acquired via an MP100WS system (BIOPAC Systems). The data were stored on a Macintosh computer, and they were analyzed by AcqKnowledge III software for the MP100WS system. The AcqKnowledge software allows for the performance of postacquisition mathematical transformations. Also, the software provides an extensive array of measurements that can be applied to the collected data. The steps involved in the quantification of anticipatory, reward, and punishment SCRs entail the following:

1. Elimination of the downdrift in the SCR wave, using a mathematical transformation function named "Difference." This function measures the difference (in amplitude) of two sample points that are separated by 10 samples. Then the difference is divided by the time interval between the first selected sample and the last selected sample.

2. Measurement of the "area under the curve" in the $5 \mathrm{sec}$ time window after a card is selected (for reward and punishment SCRs). This is the measurement of the "area under the curve" in the time window between the end of the $5 \mathrm{sec}$ after a card is clicked and before the next click of a card (for anticipatory SCRs). The "area under the curve" measurement is similar to the function of an "integral" except that, instead of using zero as a baseline for integration, a straight line is drawn between the endpoints of the selected area to function as the baseline. The area is expressed in terms of amplitude units $(\mu \mathrm{S})$ per time interval (seconds).

3 . In the case of reward and punishment SCRs, because the time interval is always $5 \mathrm{sec}$, we divide each area under the curve measurement by 5 . The area measurements per second $(\mu \mathrm{S} / \mathrm{sec})$ from all of the reward SCRs of the good decks are averaged. Averaging also is performed on all of the reward SCRs from the bad decks, all of the punishment SCRs from the good decks, and all of the punishment SCRs from the bad decks. Thus, for each subject we obtain two dependent variables of reward SCRs (from good decks and from bad decks) and two dependent variables of punishment SCRs (from good decks and from bad decks).

4. In the case of anticipatory SCRs, the time interval varies from trial to trial, but on average it is also $\sim 5 \mathrm{sec}$. Therefore, each area measurement from an individual trial is divided by its correspondent time interval. The area measurements per second $(\mu \mathrm{S} / \mathrm{sec})$ from all of the anticipatory SCRs of the good decks are averaged together as are those from the bad decks. Thus, for each subject we obtain two dependent variables of anticipatory SCRs (one from the good decks and one from the bad decks).

SCR conditioning with a loud sound. We used monochrome color slides (blue) as the conditioned stimulus (CS), a startling loud and obnoxious sound (a fog horn) as the unconditioned stimulus (US), and electrodermal activity (SCR) as the dependent measure of autonomic conditioning. Each experiment involved (1) a habituation phase in which four color stimuli (blue, red, green, orange) were presented repeatedly without the US and (2) a conditioning phase in which the blue slides were paired with the US. Six presentations of the blue slides were paired with the US, and six presentations were not; they served as test stimuli for acquiring the conditioning. The blue slides that were paired or unpaired with the US were presented at random among the other colors. Each experiment also involved (3) an extinction phase in which the blue slides were presented repeatedly without the US.

\section{RESULTS}

\section{Anatomy}

Two of the amygdala patients had suffered childhood encephalitis and later were subjected to bilateral stereotaxic amygdalotomy for the treatment of aggressive behavior. The anatomy of their lesions has been shown in previous publications (Lee et al., 1988a,b, 1995). All of the other patients were selected from the Patient Registry of the University of Iowa's Division of Behavioral Neurology and Cognitive Neuroscience; the anatomy of their lesions is presented in Figure 1.

One of the amygdala patients had congenital bilateral amygdala damage from Urbach-Wiethe disease (Tranel and Hyman, 1990), two had childhood encephalitis as indicated earlier, and the other two had herpes simplex encephalitis during adulthood. One of the VMF patients had a frontal cyst thought to have developed at age 2 years as a result of a head injury. The cyst has not been removed, and neuroimaging scans show bilateral compression of the frontal poles and the anterior ventromedial regions of the prefrontal cortex. The other four VMF patients had bilateral damage in the ventromedial sector of the frontal lobes because of meningioma or stroke. None of the patients suffered from mental retardation. All subjects (controls and patients) provided informed consent in accordance with the Human Subjects Committee of the University of Iowa.

All of the amygdala patients had lesions that involved substantial portions of the amygdala bilaterally. Two of the amygdala patients also had minimal damage to the hippocampal formation and surrounding cortices. The other three patients with amygdala lesions had damage that included the hippocampus and surrounding cortices (Fig. 1). All of the VMF patients had lesions confined to the ventral and low mesial sectors of the frontal lobe in both the right and left hemispheres (Fig. 1).

\section{Behavioral performance}

We subdivided the 100 card selections into five blocks of 20 cards each, and for each block we counted the number of selections from decks A and B (disadvantageous) and the number of selections from decks C and D (advantageous). Figure 2 represents the results as a function of group, block, and deck type. As the task progressed, normal controls gradually shifted their preference toward the good decks (C and D) and away from the bad decks (A and B). By contrast, both the amygdala and VMF patients failed to demonstrate this shift in behavior. By and large, they selected more cards from the bad decks than from the good decks. A 3 (group) $\times 2$ (deck type: good vs bad) $\times 5$ (block) ANOVA on the number of cards selected revealed significant interactions between groups and decks $\left(F_{2,20}=12.4 ; p<0.05\right)$. The interaction between groups and decks was significant when the control subjects were compared with the amygdala patients $\left(F_{1,16}=13.9 ; p<\right.$ $0.05)$ or with the VMF patients $\left(F_{1,16}=14.1 ; p<0.05\right)$, but not when the amygdala group was compared with the VMF group.

When we looked at individual performances, three subjects in the normal control group $(n=13)$ showed a disadvantageous 


\section{A}
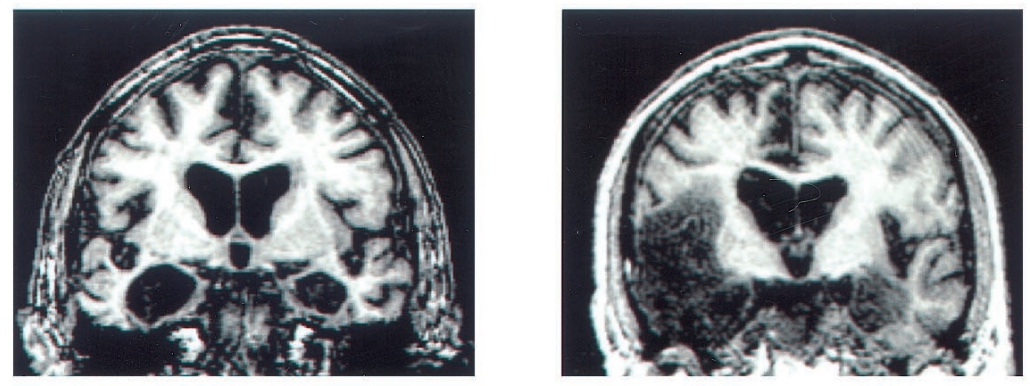

Figure 1. Neuroanatomical findings in the two groups of brain-damaged patients. $A$, Bilateral amygdala lesions. Coronal sections through the amygdala from the three patients in our Registry show complete bilateral destruction of the amygdala. The lesions from the two remaining amygdala patients have been shown in previous publications (Lee et al., 1988a,b, 1995). B, Bilateral VMF lesions. Shown are mesial and inferior views of the overlap of lesions from four VMF patients. The lesions from individual subjects were transferred onto a reference brain by using the MAP-3 technique (Frank et al., 1997). The coronal section shows an area of the ventromedial prefrontal cortex where maximum overlap occurs. The position of the cut is indicated on the brain on the left. The color bar below shows the color code corresponding to the number of overlapping lesions. The lesion of the fifth VMF patient is not part of the MAP-3 image because, as explained in the text, this patient suffered from a frontal lobe cyst at age 2 . The lack of a clear structural lesion at macroscopic level precludes the transfer into MAP-3.
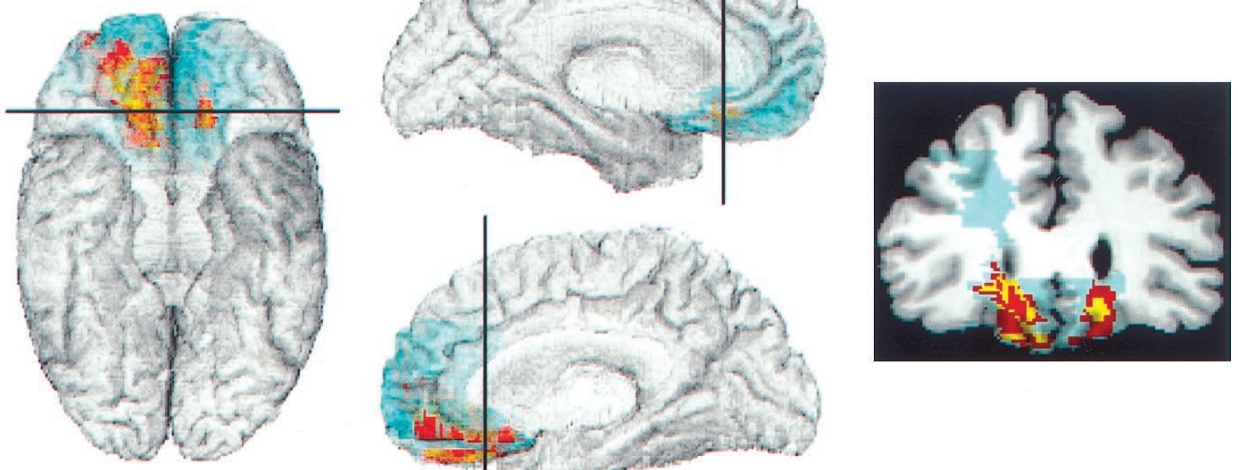

performance, in that they selected more cards from the bad decks than from the good decks. In the VMF group, however, only one patient (of five patients) behaved in an advantageous manner, choosing more cards from the good decks than from the bad decks. However, the patient still selected more cards from the bad decks (A and $\mathrm{B}$ ) than 1.6 SD above the average of cards picked from decks A and B by normal controls. All five amygdala patients behaved as predicted, choosing more cards from the bad decks than from the good decks. There was no difference in performance between the amygdala patients who acquired the damage early and those that had acquired it late in life. However, there was some difference between the patients who had damage restricted to the amygdala and those whose damage involved the hippocampus, irrespective of the time of onset of the lesion. The
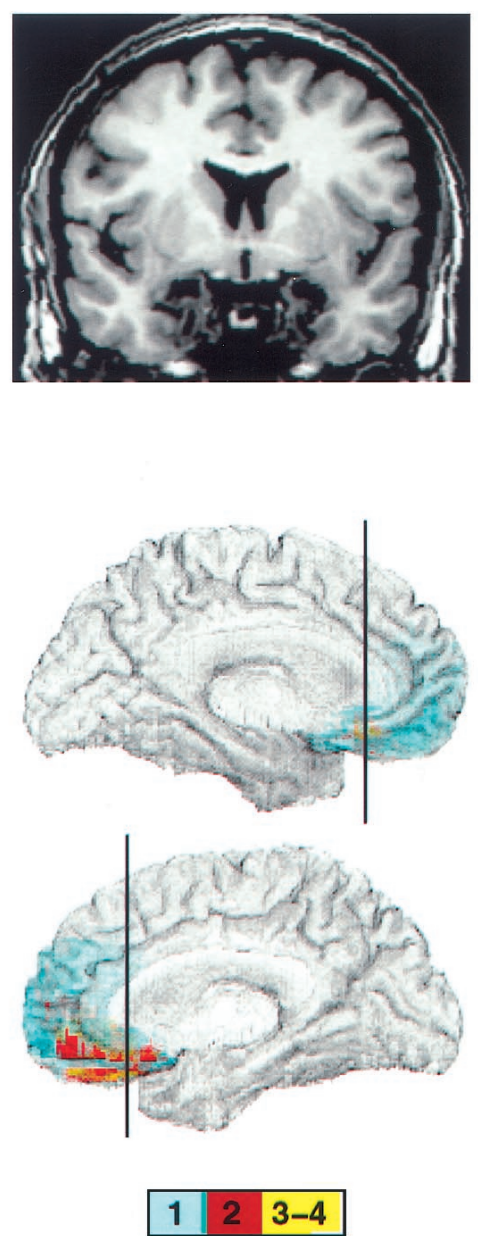

amygdala patients with hippocampal sparing performed worse than those with hippocampal damage (i.e., chose more disadvantageous cards). This observation, however, does not mean that hippocampal damage somehow improves performance on the gambling task. The better score on the gambling task of amygdala- plus hippocampus-damaged patients is the indirect consequence of the presence of an amnesic syndrome that leads the patients to make a random sampling of cards. Random sampling brings the performance score closer to 50 cards from the good decks and 50 cards from the bad decks. By contrast, the patients with only amygdala damage are similar to the VMF patients in that they are deliberate in their pursuance of a disadvantageous course of action. Their selection from the bad decks is more frequent and thus goes farther away from the 50:50 score. 


\section{Normal Control $(\mathrm{N}=13)$}

Amygdala

$(\mathbf{N}=5)$
Ventromedial Prefrontal (VMF)

$(\mathrm{N}=5)$

\section{Disadvantageous Decks (A \& B)}

\section{Advantageous Decks (C \& D)}
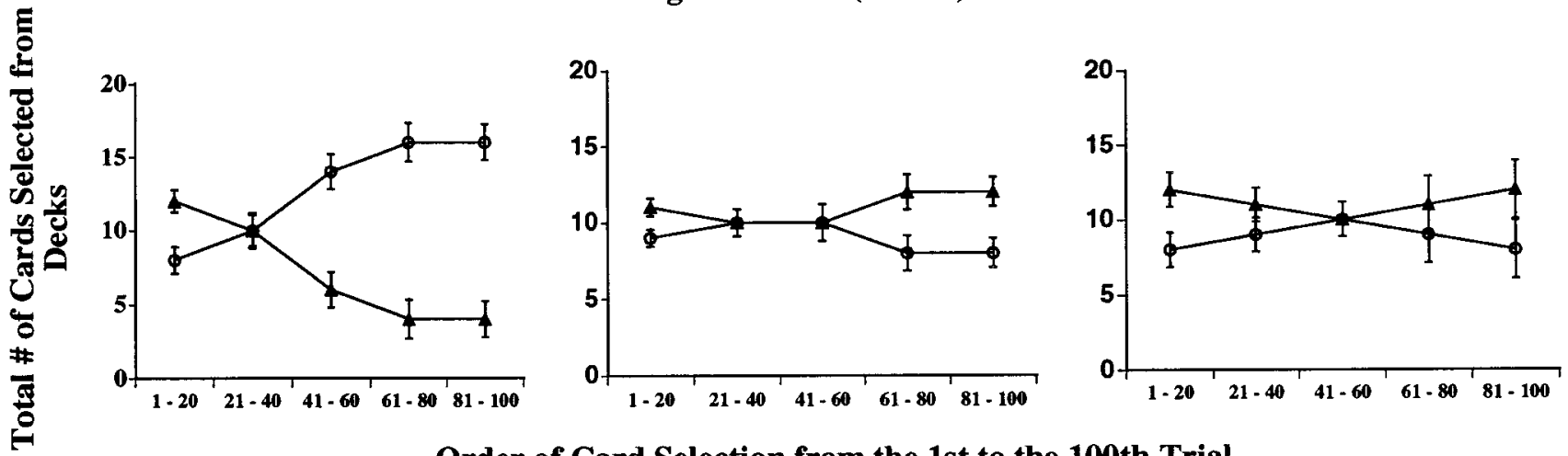

Order of Card Selection from the 1st to the 100th Trial

Figure 2. Means \pm SEM of the total number of cards selected from the advantageous versus the disadvantageous decks in each block of 20 cards, which were made by normal controls and by patients with bilateral amygdala or VMF cortex lesions. It is shown that control subjects learn to avoid the bad decks and prefer the good decks. Amygdala and VMF patients fail to do so.

\section{ANTICIPATORY SCRs}

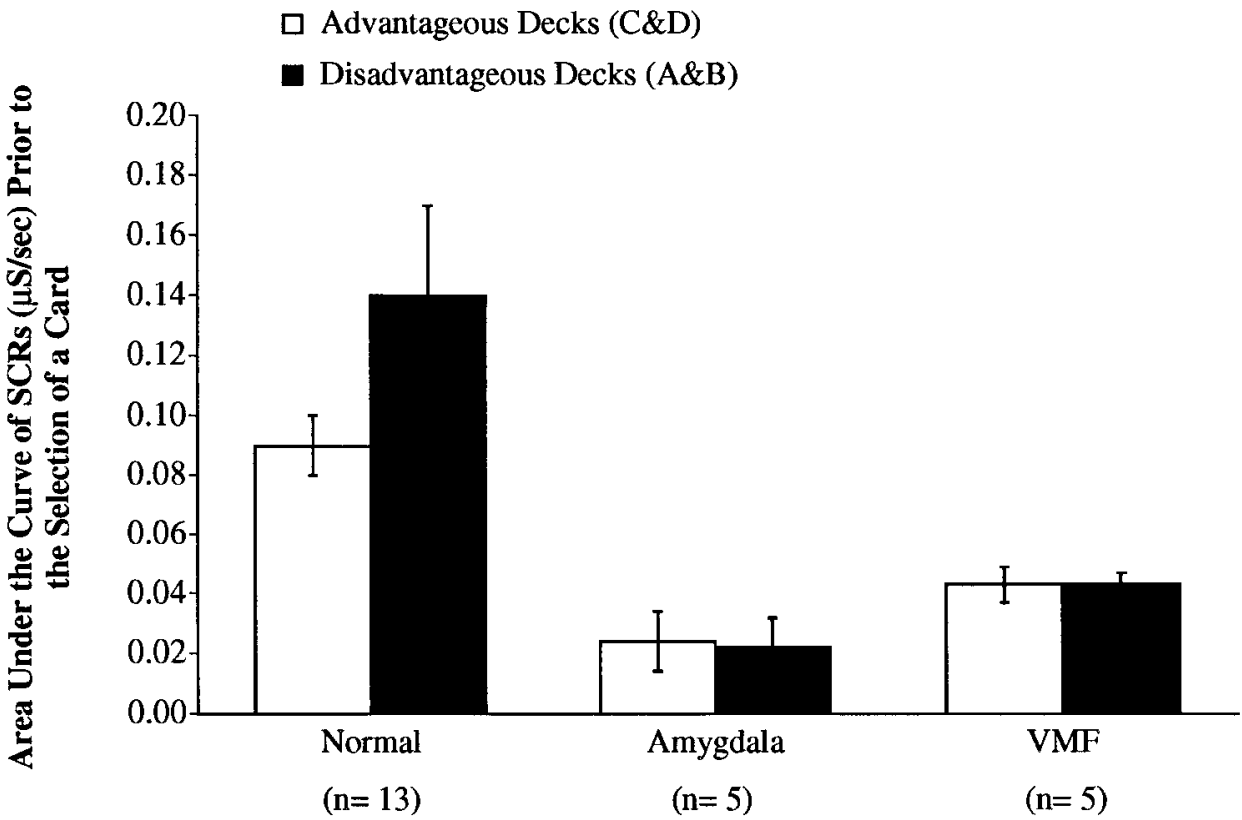

Task ABCD
Figure 3. Means \pm SEM of anticipatory SCRs $(\mu \mathrm{S} / \mathrm{sec})$ generated by controls, amygdala, or VMF patients in association with the advantageous decks $(\mathrm{C}$ and $\mathrm{D}$, white columns) versus the disadvantageous decks (A and B, black columns).

\section{Anticipatory SCRs}

Figure 3 shows that normal controls developed anticipatory SCRs. In amygdala and VMF patients these anticipatory SCRs were significantly lower in magnitude in comparison with normal controls. A 3 (group) $\times 2$ (deck type: good vs bad) ANOVA on these anticipatory SCRs revealed a significant main effect of groups $\left(F_{2,20}=5.2 ; p<0.05\right)$. Post hoc comparison of these anticipatory SCRs (Newman-Keuls) revealed significant differences between the anticipatory SCRs of controls when compared with amygdala $(p<0.05)$ or VMF $(p<0.05)$ patients, but not when the amygdala patients were compared with the VMF patients. In controls the anticipatory SCRs associated with the bad decks were significantly higher in amplitude than those associated with the good decks $(p<0.05)$. In amygdala and VMF patients no significant differences of amplitude between anticipatory SCRs from good and bad decks were seen.

Interestingly, in the three normal subjects who chose disadvantageously, the mean anticipatory SCRs from the bad decks $(0.062$ 


\section{REWARD and PUNISHMENT SCRs}

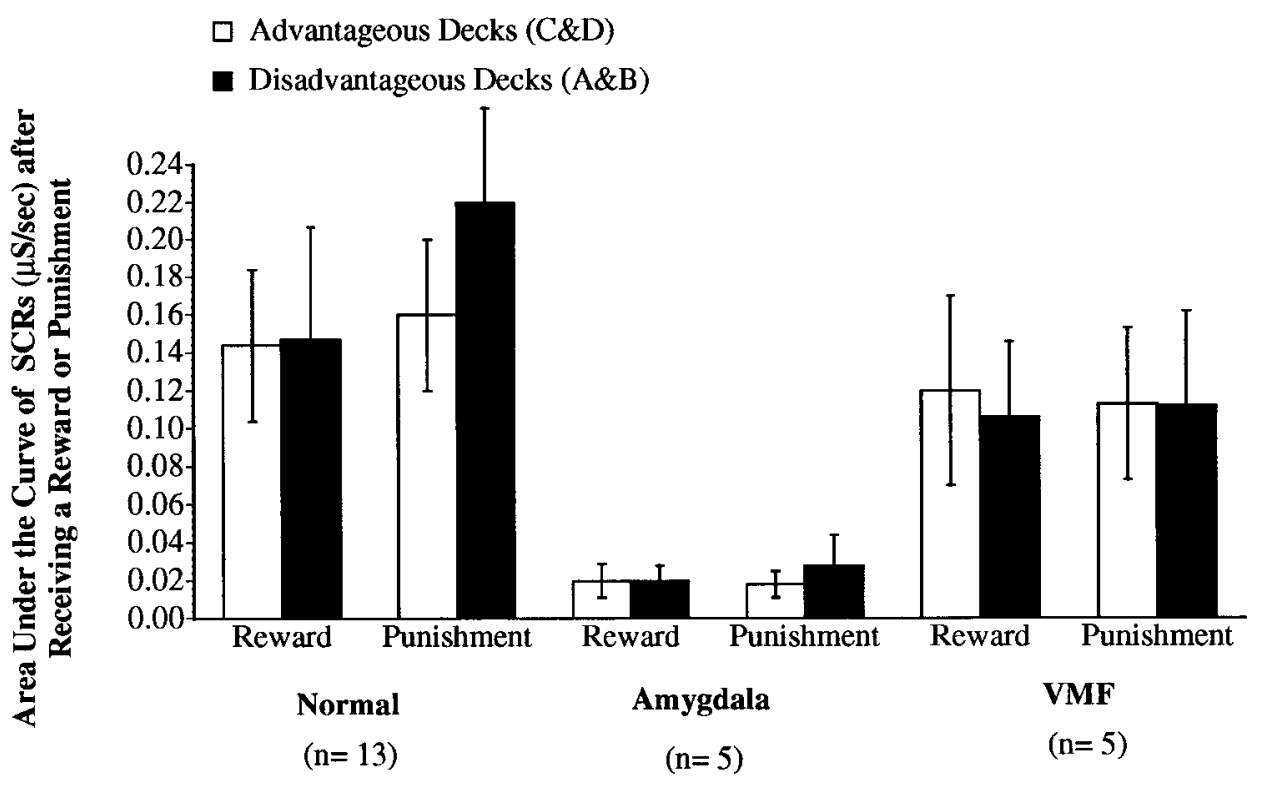

Figure 4. Means \pm SEM of reward and punishment SCRs $(\mu \mathrm{S} / \mathrm{sec})$ generated by controls, amygdala, or VMF patients in association with the advantageous decks ( $\mathrm{C}$ and $\mathrm{D}$, white columns) or the disadvantageous decks (A and B, black columns).

Task ABCD

$\mu \mathrm{S} / \mathrm{sec})$ were smaller than the mean from the good decks $(0.067$ $\mu \mathrm{S} / \mathrm{sec})$. This is quite the opposite from what happened in general in normal subjects who behaved advantageously. These subjects have a mean amplitude from the bad decks $(0.160 \mu \mathrm{S} / \mathrm{sec})$ that is higher than that from the good decks $(0.090 \mu \mathrm{S} / \mathrm{sec})$. These observations are consistent with the notion that the avoidance of the risky decks is a correlate of a significant rise in anticipatory SCRs. Most intriguing is the difference seen within normal subjects, depending on how advantageously or disadvantageously they choose.

\section{Reward and punishment SCRs}

Figure 4 shows that normal controls generated SCRs after selecting cards for which they received a reward (reward SCRs) or cards for which they received a reward and a punishment (punishment SCRs). All of the amygdala patients were impaired severely in the generation of either reward or punishment SCRs, although these same patients were able to generate SCRs in response to a loud sound (see below). However, four of the five VMF patients generated reward and punishment SCRs in the normal range. Because of the lack of homogeneity of variance between groups, these data are not amenable to parametric techniques of statistical analyses. Therefore, we used an appropriate nonparametric method for data analysis. As a group, although the SCRs from the VMF group are somewhat lower than the control group, MannWhitney $U$ tests comparing the control and VMF groups did not yield a significant difference (highest $U$ value $=30, p=0.8$; lowest $U$ value $=16, p=0.1$ ). Thus, only the amygdala patients were impaired. Mann-Whitney $U$ tests on the SCR measurements from the control and amygdala groups revealed a significant difference between the groups (highest $U$ value $=5, p=0.007$; lowest $U$ value $=1, p=0.002$ ). Similar Mann-Whitney $U$ tests on the SCR measurements from the VMF and amygdala groups revealed a significant difference between the groups (highest $U$ value $=3, p=0.047$; lowest $U$ value $=2, p=0.028$ ).

It is interesting to note that the lowering of the average reward/ punishment SCRs in the VMF group was attributable to only one patient who did not generate SCRs to reward and punishment. Interestingly, this same subject also did not acquire conditioned SCRs (see below) and thus behaved more like the patients with amygdala lesions. However, even with the inclusion of this patient the analysis comparing the VMF with the amygdala group still yielded a significant difference. This suggests that amygdala and VMF damage exerts distinct effects on the ability to generate SCRs after reward or punishment is received.

Nonetheless, it is intriguing to speculate why this patient behaved more like the amygdala than the VMF patients. We began to explore this issue with more patients. Our preliminary finding is that there may be an anatomical explanation for the difference. VMF patients who do not generate punishment and reward SCRs and do not acquire conditioned SCRs (Tranel et al., 1996) seem to have bilateral ventromedial prefrontal cortex lesions that extend more posteriorly and probably include the basal forebrain. Consistent with this observation, the VMF patient in question does indeed have a lesion that extends into the posterior region of the prefrontal cortex.

\section{Early trial versus late trial SCRs}

The SCR measures (anticipatory vs reward/punishment) obtained in our study are temporally adjacent. Although there is no evidence in the psychophysiology literature to support this possibility, we still considered the possibility that the reward/punishment SCRs observed in VMF patients were delayed anticipatory SCRs. In other words, it could be that the anticipatory SCRs in VMF patients would have a slower emergence so that they would appear at a later time window, i.e., after selecting the card rather than before. Therefore, we analyzed the data in terms of early versus late trials. The rationale for this approach was based on previous studies (Bechara et al., 1996, 1997a), which showed that the generation of anticipatory SCRs is less evident during the early trials than in the late trials. In normal controls we would expect to see a rise in anticipatory SCRs as we move from the 
A

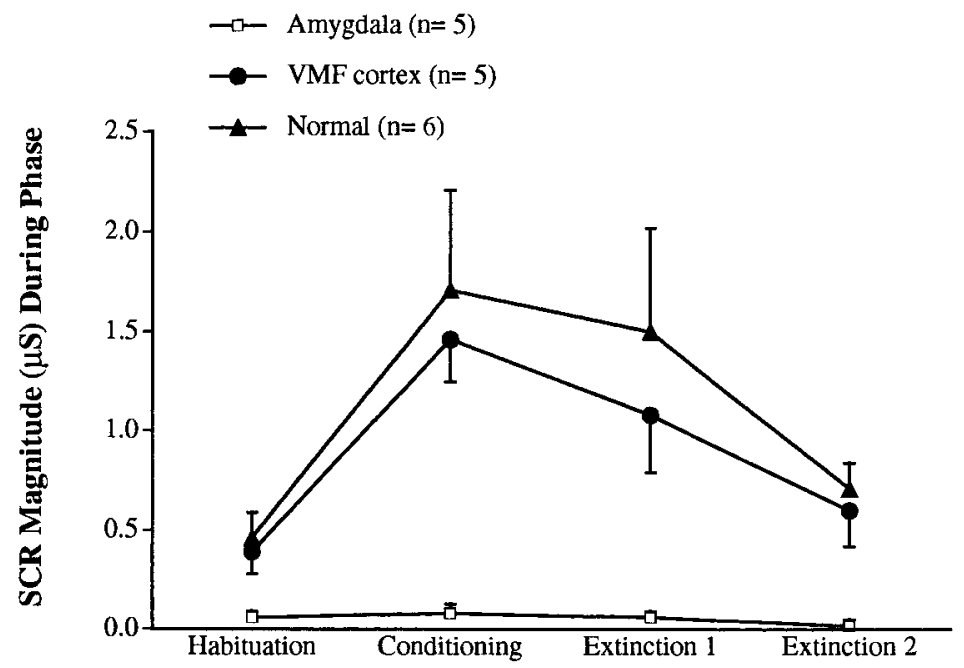

B

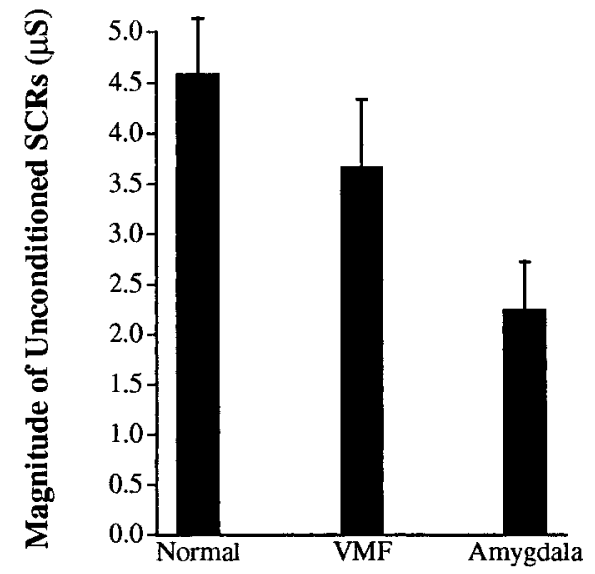

Figure 5. A, Magnitudes of SCRs in the conditioning phase as compared with the SCRs in the habituation and extinction phases. Each point on the plot represents the means \pm SEM of the magnitudes of SCRs generated by control subjects, amygdala, and VMF patients during each phase of the conditioning experiment. Each Habituation score represents the mean (from $n=6$ controls, $5 \mathrm{VMF}$, and 5 amygdala patients) of the mean magnitude of SCRs generated in response to the last three slides preceding conditioning. Each Conditioning score represents the mean of the mean magnitude of SCRs generated in response to six presentations of the CS (not followed by the US). Each Extinction 1 score represents the mean of the mean magnitude of SCRs generated in response to the first three repeated presentations of the CS during extinction. Each Extinction 2 score represents the mean of the mean magnitude of SCRs in response to the last three repeated presentations of the CS. B, Magnitudes of SCRs to the blue slides (CS) that were paired with the US during conditioning. Each column represents the mean \pm SEM of the mean magnitude of SCRs generated in response to six presentations of the CS (paired with the US) from the same control subjects and brain-damaged patients as in $A$.

early to the late trials. On the other hand, we would anticipate a slight drop in reward/punishment SCRs (because of habituation) as we move from the early to late trials. In VMF patients we would not expect a change in anticipatory SCRs, but we would expect the changes in reward/punishment SCRs to be similar to those of controls. Control and VMF groups showed similar changes in reward/punishment SCRs between the two epochs. However, in relation to the anticipatory SCRs, only the control group showed the expected change. This comparison rules out the possibility that VMF patients might have been generating delayed anticipatory SCRs.

\section{Conditioned and unconditioned SCRs}

All control subjects showed conditioning in that they began to generate SCRs after the presentation of a slide previously paired with a loud sound, and so did four of the five VMF patients. The five amygdala patients failed to show any conditioned SCRs. Figure $5 A$ shows that the conditioned SCRs generated by control subjects and VMF patients during the conditioning phase were significantly higher than those generated during the habituation or extinction phase (Newman-Keuls tests; $p$ values $<0.001$ ). The five amygdala patients did not show any signs of conditioning, and the differences were not significant ( $p$ values $>0.05$ ). Figure $5 B$ reveals that all subjects (controls, VMF, and amygdala) generated SCRs to the US (loud sound), albeit that the SCRs in the amygdala patients were lower than those in controls or VMF patients. Thus, all of the amygdala patients failed to generate SCRs to winning and losing money (in the previous experiment), and they failed to acquire the conditioning (present experiment). However, they were able to generate SCRs to a primary US such as a startling loud sound.

\section{DISCUSSION}

Our first hypothesis that the amygdala is a critical structure in a neural system necessary for somatic state activation and for implementing advantageous decisions is supported by the finding that amygdala patients failed to generate anticipatory SCRs before selecting a disadvantageous response. They also performed abnormally in the gambling task. Support for our second hypothesis, that the amygdala and VMF cortex play different roles in the process of decision-making, comes from the finding that there were differences in the profiles of impairment in the two groups despite some similarities. VMF patients did generate somatic states when told that they had won or lost play money, whereas amygdala patients failed to do so. VMF patients did acquire conditioned SCRs to a loud sound whereas amygdala patients did not. All patients, however, were capable of generating SCRs to the presentation of a physical stimulus such as a loud sound.

Decision-making is a complex process that we believe is dependent on the generation of somatic states (Damasio, 1994). The failure to evoke somatic states, as happens in both the amygdala and VMF patients, disturbs the process of making advantageous decisions. However, our findings suggest that the defective mechanism that led to a failure to generate somatic states is different in amygdala and VMF patients.

We see the impairment in decision-making after amygdala damage as an indirect consequence of the role of the amygdala in attaching affective attributes to stimuli. This interpretation is consistent with the studies showing that monkeys with lesions of the amygdala have an increased tendency to approach objects such as snakes (Kluver and Bucy, 1939; Zola-Morgan et al., 1991; 
Aggleton, 1992), as if the object of fear can no longer evoke a state of fear. This also is supported by the present and previous findings (Bechara et al., 1995; LaBar et al., 1995, 1998) that amygdala damage prevents the development of conditioned SCRs to visual stimuli paired with an aversive sound. In addition, numerous experimental studies showed that amygdala damage interferes with processing the affective attributes of reward stimuli as well. This effect has been shown in rats with food and sex reinforcement (Everitt et al., 1989; Hatfield et al., 1996; Robledo et al., 1996) and in monkeys with food reinforcement (Malkova et al., 1997). Thus, in humans, after amygdala damage the loss of money can no longer evoke the somatic state of punishment. Failure to evoke somatic states after winning or losing money would preclude the reconstitution of such somatic states when deliberating a decision with future consequences.

Not all of the amygdala patients had selective bilateral amygdala damage. Three of the patients had substantial damage to the hippocampal formation and surrounding areas. They suffered from severe anterograde memory deficit, which could be thought to influence the decision-making process. Despite these extended lesions and additional impairments, we do not believe that the decision-making impairment detected in these patients is related to the nonamygdala damage for two reasons. First, the two patients who had damage restricted to the amygdala were in fact those who exhibited the most severe behavioral impairment in the gambling task. Second, in another study we tested a group of amnesic patients suffering from anoxic encephalopathy, which is known to damage CA1 cells of the hippocampus rather than the amygdala (Zola-Morgan et al., 1986; Rempel-Clower et al., 1996). We found that, although amnesiacs do not perform as well as normal controls in the gambling task, they still make predominantly advantageous choices (Bechara et al., 1997b). Therefore, it is unlikely that the hippocampal damage in our amygdala patients could be responsible for the findings of impairment in the gambling task.

The notion that bilateral damage to the amygdala is associated with decision-making impairments in the gambling task also is supported by the observation that amygdala patients demonstrate poor judgment and decision-making in their real-life social behavior (Tranel and Hyman, 1990; Adolphs et al., 1995). Furthermore, some amygdala patients show an inability to evoke somatic states after winning or losing in real-life settings (Damasio et al., 1985).

Unlike the amygdala patients, the VMF patients did acquire the SCR conditioning with an aversive loud sound, and they did generate SCRs when they won and lost money in the gambling task. This finding is consistent with the conditioning studies in animals showing that the VMF cortex is not necessary for the acquisition of fear conditioning (Morgan and LeDoux, 1995). Similarly, the human VMF cortex, especially its anterior compartment, is not necessary for conditioning involving the association of a stimulus with a primary unconditioned stimulus such as an aversive loud sound (Tranel et al., 1996). This indicates that, unlike the amygdala, the VMF cortex is not necessary for mediating the affective attributes of a stimulus charged with emotion. However, we concede that the VMF cortex may play some role in this process. Indeed, previous work with VMF patients showed that the patients failed to generate SCRs to emotionally charged pictures when they viewed these pictures passively (Damasio et al., 1990). However, the same patients generated normal magnitude SCRs to the same target pictures when they were asked to view and describe the content of the pictures (Damasio et al., 1990). The results suggest that the patients may have a weakened ability to process the affective attribute of an emotional stimulus. Perhaps this could explain the slightly lower magnitude SCRs generated by VMF patients after receiving reward or punishment, relative to normal control subjects (see Fig. 4). Despite such a possible weakness, the results show that the VMF patients are not impaired in their ability to generate SCRs to emotionally significant events. This stands in contrast to the amygdala patients who are severely impaired in this domain.

We suggest that the mechanism underlying the decisionmaking impairment associated with VMF damage is more complex than that of the amygdala. After the somatic states of reward and punishment are evoked with individual card draws, each deck becomes associated with numerous and conflicting states of reward and punishment. The role of the VMF cortex comes into play when subjects sort out this conflict and decide whether to seek or avoid the deck. The poor decision-making associated with VMF damage is related to an inability to integrate effectively all of the somatic state information triggered by the amygdala as well as other somatic effectors such as the hypothalamus and brainstem nuclei. Indeed, the VMF cortex has extensive bi-directional connections with the amygdala (Amaral and Price, 1984; Van Hoesen, 1985; Amaral et al., 1992). When subjects decide to select cards from a specific deck, the neural activity pertaining to this information is signaled to VM cortices, which in turn activate the amygdala (Damasio et al., 1991). This latter activity would reconstitute a somatic state that integrates the numerous and conflicting instances of reward and punishment encountered with individual card draws from that deck. In the end, if the negative somatic states outweigh the positive ones, an overall negative state is enacted and is indexed by the anticipatory SCRs we observed before the selection of cards from the disadvantageous decks. In turn, this influences the decision to avoid the deck under consideration.

It is important to note that SCRs are viewed by psychophysiologists as a measure of only general arousal (Venables and Christie, 1975). Our SCR measures do not necessarily distinguish between positive and negative somatic states. This distinction, however, is not relevant to the goals of this study. Indeed, our punishments SCRs (see Fig. 4) are not pure responses to punishment. Each of these SCRs was a response to a reward, followed by a punishment (e.g., you won an $\mathrm{X}$ amount... but you lost a $\mathrm{Y}$ amount). Furthermore, SCRs are more sensitive to negative than positive states (Venables and Christie, 1975). Therefore, it is likely that the anticipatory SCRs we see in normal subjects (see Fig. 3) reflect increased arousal to the higher losses in the disadvantageous decks.

The current study parallels the Schoenbaum et al. (1998) study in animals suggesting that both the orbitofrontal cortex and basolateral amygdala provide a critical circuit for the learning that underlies goal-directed behavior (Schoenbaum et al., 1998). Our finding is significant because the nature of the deficit revealed after VMF or amygdala damage may reflect two types of decision-making deficits observable in the behaviors of real-life activities of these patients. The decision-making impairments of patients with VMF cortex lesions have remote consequences and usually do not cause bodily harm. For instance, VMF patients make choices that lead to long-term financial losses or to the loss of friend and family relationships down the line (Eslinger and Damasio, 1984), but they never engage in actions that may lead to physical harm to themselves or to others. On the other hand, although patients with bilateral amygdala lesions do exhibit decision-making impairments in the social realm similar to those of the VMF patients (Tranel and Hyman, 1990; Adolphs et al., 
1995), they actually can pursue actions that eventually lead to physical harm to themselves and to others. Indeed, with one exception (Adolphs et al., 1995), amygdala patients who participated in this study live under supervised care and are unable to function alone in society. In two of the cases the patients have pursued actions that endangered themselves and others (Lee et al., 1988a,b, 1995).

\section{REFERENCES}

Adolphs R, Tranel D, Damasio H, Damasio AR (1995) Fear and the human amygdala. J Neurosci 15:5879-5892.

Aggleton JP (1992) The functional effects of amygdala lesions in humans: a comparison with findings from monkeys. In: The amygdala: neurobiological aspects of emotion, memory, and mental dysfunction (Aggleton JP, ed), pp 485-504. New York: Wiley-Liss.

Amaral DG, Price JL (1984) Amygdalo-cortical connections in the monkey (Macaca fascicularis). J Comp Neurol 230:465-496.

Amaral DG, Price JL, Pitkanen A, Carmichael ST (1992) Anatomical organization of the primate amygdaloid complex. In: The amygdala: neurobiological aspects of emotion, memory, and mental dysfunction (Aggleton JP, ed), pp 1-66. New York: Wiley-Liss.

Bechara A, Damasio AR, Damasio H, Anderson SW (1994) Insensitivity to future consequences following damage to human prefrontal cortex. Cognition 50:7-15.

Bechara A, Tranel D, Damasio H, Adolphs R, Rockland C, Damasio AR (1995) Double dissociation of conditioning and declarative knowledge relative to the amygdala and hippocampus in humans. Science 269:1115-1118.

Bechara A, Tranel D, Damasio H, Damasio AR (1996) Failure to respond autonomically to anticipated future outcomes following damage to prefrontal cortex. Cereb Cortex 6:215-225.

Bechara A, Damasio H, Tranel D, Damasio AR (1997a) Deciding advantageously before knowing the advantageous strategy. Science 275:1293-1295.

Bechara A, Tranel D, Damasio H, Damasio AR (1997b) An anatomical system subserving decision-making. Soc Neurosci Abstr 23:495.

Bechara A, Damasio H, Tranel D, Anderson SW (1998) Dissociation of working memory from decision making within the human prefrontal cortex. J Neurosci 18:428-437.

Damasio AR (1994) Descartes' error: emotion, reason, and the human brain. New York: Grosset/Putnam.

Damasio AR (1996) The somatic marker hypothesis and the possible functions of the prefrontal cortex. Philos Trans R Soc Lond [Biol] 351:1413-1420.

Damasio H, Damasio AR (1989) Lesion analysis in neuropsychology. New York: Oxford UP.

Damasio H, Frank R (1992) Three-dimensional in vivo mapping of brain lesions in humans. Arch Neurol 49:137-143.

Damasio AR, Eslinger PJ, Damasio H, Van Hoesen GW, Cornell S (1985) Multimodal amnesic syndrome following bilateral temporal and basal forebrain damage. Arch Neurol 42:252-259.

Damasio AR, Tranel D, Damasio H (1990) Individuals with sociopathic behavior caused by frontal damage fail to respond autonomically to social stimuli. Behav Brain Res 41:81-94.

Damasio AR, Tranel D, Damasio H (1991) Somatic markers and the guidance of behavior: theory and preliminary testing. In: Frontal lobe function and dysfunction (Levin HS, Eisenberg HM, Benton AL, eds), pp 217-229. New York: Oxford UP.

Eslinger P, Damasio AR (1984) Behavioral disturbances associated with rupture of anterior communicating artery aneurysms. Semin Neurol 4:385-389.

Everitt BJ, Cador M, Robbins TW (1989) Interactions between the amygdala and ventral striatum in stimulus-reward associations: studies using a second-order schedule of sexual reinforcement. Neuroscience 30:63-75.

Frank R, Damasio H, Grabowski TJ (1997) Brainvox: an interactive multimodal visualization and analysis system for neuroanatomical imaging. NeuroImage 5:13-30.

Hatfield T, Han JS, Conley M, Gallagher M, Holland P (1996) Neurotoxic lesions of basolateral, but not central, amygdala interfere with Pavlovian second-order conditioning and reinforcer devaluation effects. J Neurosci 16:5256-5265.

Kluver H, Bucy PC (1939) Preliminary analysis of functions of the temporal lobes in monkeys. Arch Neurol Psychiatry 42:979-997.

LaBar KS, LeDoux JE, Spencer DD, Phelps EA (1995) Impaired fear conditioning following unilateral temporal lobectomy in humans. J Neurosci 15:6846-6855.

LaBar KS, Gatenby JC, Gore JC, LeDoux JE, Phelps EA (1998) Human amygdala activation during conditioned fear acquisition and extinction: a mixed-trial fMRI study. Neuron 20:937-945.

Lee GP, Arena JG, Meador KJ, Smith JR, Loring DW, Flanigin HF (1988a) Changes in autonomic responsiveness following bilateral amygdalotomy in humans. Neuropsychiatry Neuropsychol Behav Neurol 1:119-129.

Lee GP, Meador KJ, Smith JR, Loring DW, Flanigin HF (1988b) Preserved crossmodal association following bilateral amygdalotomy in man. Int J Neurosci 40:47-55.

Lee GP, Reed MF, Meador KJ, Smith JR, Loring DW (1995) Is the amygdala crucial for cross-modal association in humans? Neuropsychologia 9:236-245.

Malkova L, Gaffan D, Murray EA (1997) Excitotoxic lesions of the amygdala fail to produce impairment in visual learning for auditory secondary reinforcement but interfere with reinforcer devaluation effects in rhesus monkeys. J Neurosci 17:6011-6020.

Morgan MA, LeDoux JE (1995) Differential contribution of dorsal and ventral medial prefrontal cortex to the acquisition and extinction of conditioned fear in rats. Behav Neurosci 109:681-688.

Rempel-Clower NL, Zola SM, Squire LR, Amaral DG (1996) Three cases of enduring memory impairment after bilateral damage limited to the hippocampal formation. J Neurosci 16:5233-5255.

Robledo P, Robbins TW, Everitt BJ (1996) Effects of excitotoxic lesions of the central amygdaloid nucleus on the potentiation of reward-related stimuli by intra-accumbens amphetamine. Behav Neurosci 110:981-990.

Schoenbaum G, Chiba AA, Gallagher M (1998) Orbitofrontal cortex and basolateral amygdala encode expected outcomes during learning. Nat Neurosci 1:155-159.

Tranel D, Hyman BT (1990) Neuropsychological correlates of bilateral amygdala damage. Arch Neurol 47:349-355.

Tranel D, Bechara A, Damasio H, Damasio AR (1996) Fear conditioning after ventromedial frontal lobe damage in humans. Soc Neurosci Abstr 22:1108.

Van Hoesen GW (1985) Neural systems of the non-human primate forebrain implicated in memory. Ann NY Acad Sci 444:97-112.

Venables PH, Christie MJ (1975) Mechanisms, instrumentation, recording techniques, and quantification of responses. In: Electrodermal activity in psychological research (Prokasy WJ, Raskin DC, eds), pp 1-124. New York: Academic.

Zola-Morgan S, Squire LR, Amaral DG (1986) Human amnesia and the medial temporal region: enduring memory impairment following a bilateral lesion limited to field CA1 of the hippocampus. J Neurosci 6:2950-2967.

Zola-Morgan S, Squire LR, Alverez-Royo P, Clower RP (1991) Independence of memory functions and emotional behavior: separate contributions of the hippocampal formation and the amygdala. Hippocampus 1:207-220. 